\title{
Advantages of Endoscope-Assisted Disc-FX in Surgical Management of Lumbar Disc Herniation: A Report of 10 Cases
}

Hirano $\mathbf{Y}^{1}$, Pawar $\mathbf{S}^{\mathbf{1}, 2}$, Bansil $\mathbf{R}^{\mathbf{1 , 3}}$ and Watanabe $\mathbf{K}^{\mathbf{1}}$

${ }^{1}$ Department of Neurosurgery, Southern Tohoku Research Institute for Neuroscience, Koriyama, Fukushima, Japan

${ }^{2}$ Department of Paediatric Neurosurgery, Narayana Health-SRCC Children's Hospital, Mumbai, Maharashtra, India

${ }^{3}$ Department of Neurosurgery, Institute of Neurosciences, BLK Super-specialty Hospital, New Delhi, India

\begin{abstract}
Objective: Microsurgical discectomy is the gold standard treatment for the herniated nucleus pulposus (HNP) of the lumbar spine. On the other hand, less invasive procedures have been developed and accepted rapidly. Percutaneous endoscopic lumbar discectomy (PELD) was developed as one of the minimally invasive techniques for HNP of the lumbar spine, and satisfactory outcomes have been reported. For the contained type HNP or discogenic pain, however, surgical indication of PELD is sometimes controversial. We describe our experiences of surgical treatment with Disc$\mathrm{FX}$, focusing on advantages of endoscopic observation.
\end{abstract}

Patients and Methods: A total of 10 patients with contained type HNP were treated with Disc-FX system. DiscFX was carried out percutaneously under local anesthesia using a cannula of $3.4 \mathrm{~mm}$ diameter, with a trajectory compatible with the transforaminal approach of the PELD. Endoscope was introduced intermittently in 5 patients to observe the results of each procedure.

Results: Sufficient pain relief was achieved in all patients, and Japanese Orthopedic Association score improved from 7-16 (mean 13.0) to 22-29 (mean 25.9), and visual analogue scale improved from 6-10 (mean 8.0) to 0-3 (mean 1.2). Endoscopic observation enabled the procedure almost equivalent to targeted fragmentectomy, and contributed in better surgical outcome.

Conclusion: Disc-FX is a promising procedure in the armamentarium of minimally invasive surgery for the selected conditions of patients, and endoscopic observation is considered to be beneficial in more accurate and adequate decompression.

Keywords: Lumbar disc herniation; Minimally invasive surgery; Disc-FX; Endoscopic observation

\section{Introduction}

Microsurgical discectomy is the gold standard treatment for the herniated nucleus pulposus (HNP) of the lumbar spine. Conventional microsurgery is associated with good results, but some damage is caused to the surrounding musculoskeletal structure. To achieve good results with less invasiveness, percutaneous posterolateral nucleotomy was introduced in the early 1970s [1-3]. The initial concept was central disc decompression, but the surgical techniques have evolved during the subsequent 3 decades into targeted fragmentectomy [4]. The term percutaneous endoscopic lumbar discectomy (PELD) is frequently used today to describe various techniques including targeted fragmentectomy. This percutaneous surgical procedure can adopt the transforaminal [5-7], extraforaminal [8], or interlaminar approaches $[6,7,9]$. Satisfactory success rates ( $85 \%$ to $92 \%)$ have been reported, and the advantages of this minimally invasive procedure have been reported [4-13].

Surgical indication for contained type HNP and discogenic pain is controversial $[14,15]$. There may be no indication of conventional microsurgical discectomy, and application of PELD may also be questionable. But the patients complain of persistent radiculopathy and low back pain despite any kind of conservative therapy, and this condition affects their quality of life. For these conditions, the radiofrequency treatment, Disc-FX (elliquence, New York, USA), is a promising option to provide solutions [16-20]. Disc-FX is carried out percutaneously under local anesthesia using a cannula of 3.4 $\mathrm{mm}$ diameter, with a flat horizontal trajectory compatible with the transforaminal approach of the PELD. This technique allows:

- Partial nucleotomy with $2.9 \mathrm{~mm}$ grasping forceps.
- Nucleus ablation with Trigger-Flex bipolar turbo mode.

- Annulus modulation with Trigger-Flex bipolar hemo mode.

- Intermittent endoscopic observation is also possible, and similar result as targeted fragmentectomy may be expected with assistance of endoscopic observation.

We report our surgical experiences with Disc-FX, and advantages of intermittent endoscopic observation are discussed with some review of the pertinent literature.

\section{Patients and Methods}

Since December 2013, a total of 10 patients were treated by Disc-FX system in the Southern Tohoku Research Institute for Neuroscience. There were 8 male and 2 female patients, with age 30-72 years (mean 47.2 years). Affected levels were L4-L5 in 6 patients, L5-S1 in 2 patients, and 1 patient each at L3-L4 and L1-L2. Operations were carried out under local anesthesia ( $10 \mathrm{ml}$ of $1 \%$ lidocaine), and followed standard procedure (debulking, nucleus ablation, and annulus modulation) under fluoroscopy. Endoscope of $2 \mathrm{~mm}$ diameter and straight view was introduced intermittently in 5 patients to observe the results of each

*Corresponding author: Yoshitaka Hirano, Department of Neurosurgery, Southern Tohoku Research Institute for Neuroscience, Koriyama, Fukushima, Japan, Tel: +81-24-934-5322; E-mail: mth10yhirano@flute.ocn.ne.jp

Received August 24, 2018; Accepted September 06, 2018; Published September 07, 2018

Citation: Hirano Y, Pawar S, Bansil R, Watanabe K (2018) Advantages of Endoscope-Assisted Disc-FX in Surgical Management of Lumbar Disc Herniation: A Report of 10 Cases. J Spine 7: 015. doi: 10.0142/2165-7939.1000015

Copyright: @ 2018 Hirano Y, et al. This is an open-access article distributed under the terms of the Creative Commons Attribution License, which permits unrestricted use, distribution, and reproduction in any medium, provided the original author and source are credited. 
procedure. Surgical outcome was assessed at 6 months postoperatively with the Japanese Orthopedic Association (JOA) score and the visual analogue scale (VAS). All patients underwent magnetic resonance (MR) studies immediately after the operation to evaluate the accuracy of the surgical procedure.

\section{Results}

Sufficient pain relief was achieved in all patients, and the JOA score improved from 7-16 (mean 13.0) to 22-29 (mean 25.9), and VAS improved from 6-10 (mean 8.0) to 0-3 (mean 1.2). Endoscopic observation enabled the procedure almost equivalent to targeted fragmentectomy, and contributed in better surgical outcome. The MR study immediately after the operation showed adequate decompression in all the patients. There was no surgical complication, and no patient has required additional microsurgical decompression to date. In 3 patients with follow up period of more than 3 years, favourable surgical outcomes were maintained with minimum symptom of dysesthesia.

\section{Illustrative Case}

A 35-year-old man had suffered from low back pain and dysesthesia of the lower extremities for 2 months, which were resistant to any kind of conservative therapy. He visited our outpatient service, and femoral nerve stretching test was positive bilaterally. There was no muscle weakness or pathological reflexes. The magnetic resonance (MR) study (Figure 1) revealed a relatively small disc herniation at the right L3-L4 level and some disc degeneration at the L4-L5 level. The patient asked for surgical treatment due to the prolonged pain, but microsurgical discectomy or PELD were considered too much for this condition. We decided to undertake an operation with the Disc-FX system, and the entry point was decided by measuring under fluoroscopy to obtain a flat horizontal cannula trajectory, which was $12 \mathrm{~cm}$ to the right side from the midline in this patient. L3-L4 discography was carried out as the first step of the surgical procedure (Figures $2 a$ and $2 b$ ) and followed by partial nucleotomy with $2.9 \mathrm{~mm}$ grasping forceps (Figure 2c) as the debulking procedure of the L3-L4 disc space. An endoscope of 2 $\mathrm{mm}$ diameter with straight view was introduced to reveal insufficient decompression (Figure 3), and further nucleotomy, nucleus ablation

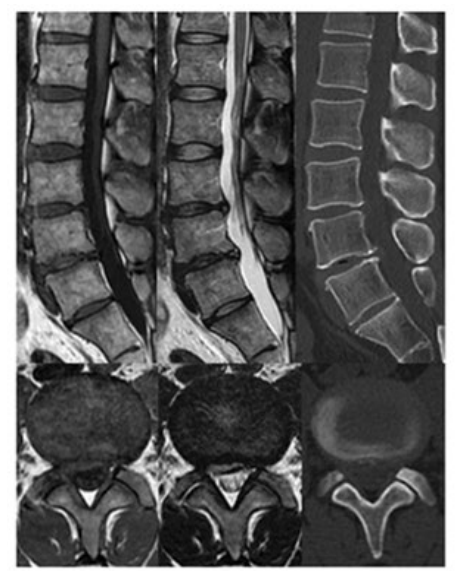

\begin{tabular}{l|l|l}
$a$ & $c$ & $e$ \\
\hline$b$ & $d$ & $f$
\end{tabular}

Figure 1: Illustrative case, a 35-year-old man presented with prolonged low back pain and dysesthesia of the lower extremities. Magnetic resonance (MR) study (a) T1-weighted midsagittal image, (b) T1-weighted axial section at the L3L4 level, (c) T2-weighted midsagittal image, and (d) T2-weighted axial section at the L3-L4 level, respectively) shows right-dominant disc herniation at the L3-L4 level, with some degenerative change at the L4-L5 level. Computerized tomography (e) reconstructed mid-sagittal section and (f) axial section at the L3-L4 level) shows no bony pathology at the L3-L4 level.
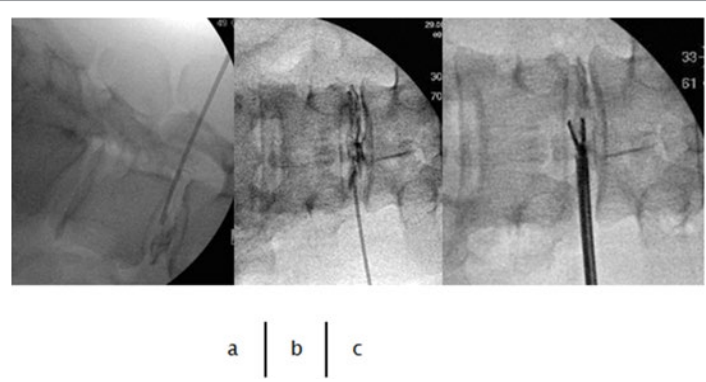

Figure 2: (a) Intraoperative fluoroscopic images showing L3-L4 discography lateral image and (b) Antero-posterior image as the first step of the Disc-FX system. (c) Debulking with the disc forceps was carried out under fluoroscopic observation.

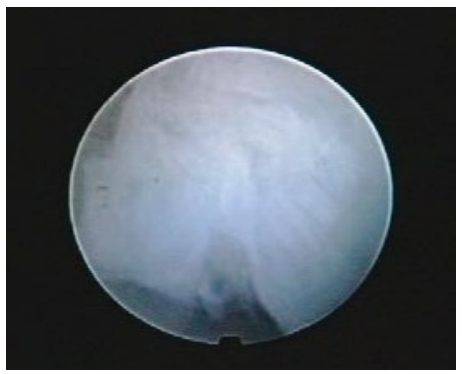

Figure 3: Endoscope of $2 \mathrm{~mm}$ diameter and straight view was introduced to reveal insufficient removal of the disc fragment.

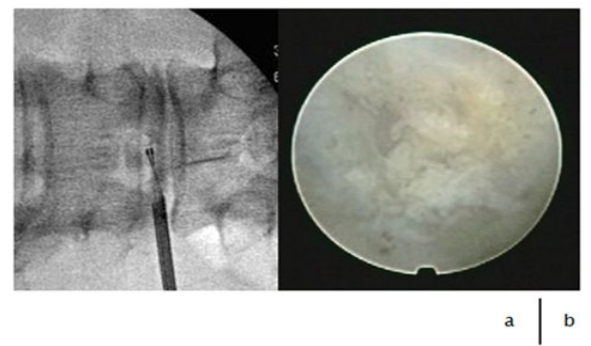

Figure 4: The cannula of the Disc-FX was moved towards dorsolateral direction to obtain a flat horizontal trajectory. Additional discectomy, ablation of the residual nucleus pulposus, and annulus modulation (a) were carried out under fluoroscopy. The final endoscopic observation (b) shows adequate decompression to confirm the end point of the surgical procedure.
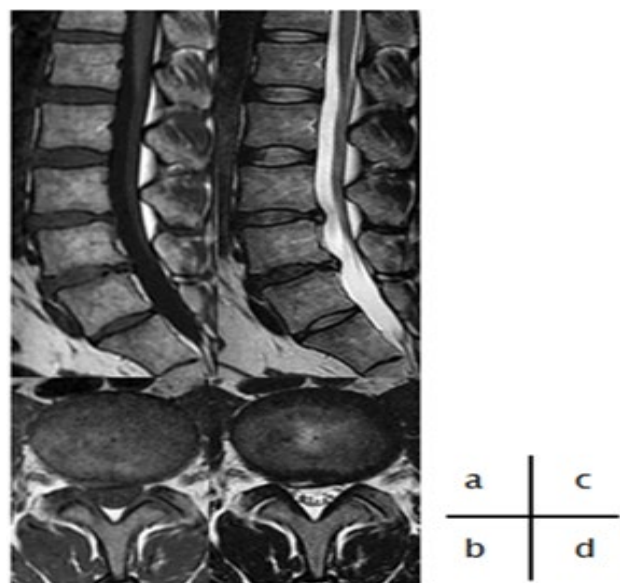

Figure 5: The MR study immediately after the operation (a) T1-weighted midsagittal image, (b) T1-weighted axial section at the L3-L4 level, (c) T2weighted midsagittal image, and (d) T2-weighted axial section at the L3-L4 level, respectively, showing adequate decompression at the L3-L4 level. 
and after moving the cannula to the dorsolateral direction, annulus modulation (Figure 4a) were carried out under fluoroscopy, assisted by intermittent endoscopic observation. Final endoscopic view (Figure 4b) confirmed satisfactory decompression and ablation. The patient became symptom free on the day of the operation, and resumed his daily activities. The MR study immediately after the operation (Figure 5) showed adequately completed procedure almost equivalent to targeted fragmentectomy at the L3-4 level.

\section{Discussion}

The treatment strategies for HNP of the lumbar spine should be primarily conservative and over $90 \%$ of the patients show satisfactory response. When the patients do not respond to any kind of conservative therapy, the current gold standard is microsurgical discectomy, and attempts to minimize surgical invasiveness, such as procedures with tubular retractors, have been proposed. However these less invasive procedures are associated with tendency of earlier surgical intervention and significant increase of costs as a result. Peul and co-authors evaluated the cost efficacy of early surgery to be an extremely expensive method from a healthcare economic perspective [21]. The complication rate as high as $8 \%$ and trauma caused by an approach are important factors in assessment of the value of surgical treatment [18]. To achieve good results with less invasiveness, percutaneous postero-lateral nucleotomy was introduced in the early 1970s [1-3]. Evolution of the spinal endoscope allowed direct visualization via a uniportal approach, and further modification allowed multichannel irrigation, specialized cannulas and discectomy tools [22,23]. Full-endoscopic surgery for $\mathrm{HNP}$ of the lumbar spine has been associated with equivalent surgical outcome as the conventional procedure and minimized surgical invasiveness [4-13], and it attracts spine surgeons today.

The Disc-FX system was developed in the hope of addressing the unsatisfactory clinical outcomes for contained type HNP, combining the knowledge gained from uniportal percutaneous approach such as nucleoplasty and the proven positive effects of high radiofrequency [18]. Through a stab wound of $4 \mathrm{~mm}$ and cannula of $3.4 \mathrm{~mm}$ diameter, this surgical procedure under local anesthesia allows:

- Debulking of the disc space with $2.9 \mathrm{~mm}$ grasping forceps.

- Nucleus ablation with Trigger-Flex bipolar turbo mode.

- Annulus modulation with Trigger-Flex bipolar hemo mode.

The use of higher radiofrequency of $1.7 \mathrm{MHz}$ through the elliquence Surgi-Max generator offers the advantage of reduced heat and minimal tissue ablation $[16,17]$.

As shown in the full-endoscopic surgery, combination of different steps improves the outcome, and additional step of intermittent endoscopic observation is expected to improve accuracy of the procedure [17]. Since targeted fragmentectomy is the goal of the current surgical treatment for lumbar disc herniation, use of endoscope is considered to be beneficial in achieving better surgical outcome. Additionally, the surgical procedure under local anaesthesia offers the patients diminished peri- and postoperative stress, and a faster recovery, mobilization, less postoperative pain and reduced medical costs are expected $[18,24]$. Furthermore, this procedure is expected to provide long-lasting pain relief, up to 2 years or more after the operation [20].

\section{Conclusion}

In conclusion, Disc-FX is a promising procedure of minimally invasive surgery for the selected conditions of patients, and endoscopic observation is considered to be beneficial to achieve more accurate and adequate decompression. This technique is significantly beneficial in providing the patients an option of avoiding an open surgery and leaving the dorsal approach virgin [18]. Current ideal indication criteria for Disc-FX would be

- Patients with small, contained type HNP.

- Patients with back pain due to annular tear.

- Patients with difficulty in accurate diagnosis due to the discrepancy between the neurological evaluation and radiological findings.

This article describes our preliminary results with a prototype endoscope, and the image quality may not be good enough to convince the advantages of endoscopic observations. With development of endoscope with higher visual quality, further improvement will be expected as the evidence on the efficacy of the Disc-FX are collected by larger studies and longer follow up.

\section{Conflict of Interest}

The authors report no conflict of interest concerning the materials or methods used in this study or the finding specified in this paper.

\section{References}

1. Hijikata S, Yamagishi M, Nakayama T, Oomori K (1975) Percutaneous nucleotomy: A new treatment method for lumbar disc herniation. Tokyo Denryoku Byoin Iho 5: 39-44

2. Kambin P, Gellman H (1983) Percutaneous lateral discectomy of the lumbar spine: A preliminary report. Clin Orthop 174: 127-132.

3. Maroon JC, Onik G, Sternau L (1989) Percutaneous automated discectomy: A new approach to lumbar surgery. Clin Orthop 238: 64-70.

4. Choi G, Lee SH, Lokhande P, Kong BJ, Shim CS, et al. (2008) Percutaneous endoscopic approach for highly migrated intracanal disc herniations by foraminoplastic techniques using rigid working channel endoscope. Spine (Phila Pa 1976) 33: E508-E515.

5. Lew SM, Mehalic TF, Fagone KL (2001) Transforaminal percutaneous endoscopic discectomy in the treatment of far-lateral and foraminal lumbar disc herniations. J Neurosurg 94(2 Suppl): 216-220.

6. Ruetten S, Komp M, Merk H, Godolias G (2007) Use of newly developed instruments and endoscopes: full-endoscopic resection of lumbar disc herniations via the interlaminar and lateral transforaminal approach. J Neurosurg Spine 6: 521-530.

7. Ruetten S, Komp M, Merk H, Godolias G (2009) Recurrent disc herniation after conventional discectomy: a prospective, randomized study comparing full-endoscopic interlaminar and transforaminal versus microsurgical revision. $J$ Spinal Disord Tech 22: 122-129.

8. Choi G, Lee SH, Bhanot A, Raiturker PP, Chae YS (2007) Percutaneous endoscopic discectomy for extraforaminal lumbar disc herniations: Extraforaminal targeted fragmentectomy technique using working channel endoscope. Spine (Phila Pa 1976) 32: E93-E99.

9. Choi G, Lee SH, Raiturker PP, Lee S, Chae YS (2006) Percutaneous endoscopic interlaminar discectomy for intracanalicular disc herniations at L5 S1 using a rigid working channel endoscope. Neurosurgery 2006; 58(1 Suppl): ONS59-ONS68.

10. Tsou PM, Yeung CA, Yeung AT (2004) Posterolateral transforaminal selective endoscopic discectomy and thermal annuloplasty for chronic lumbar discogenic pain: A minimal access visualized intradiscal surgical procedure. Spine J 4 564-573.

11. Yeung AT, Yeung CA (2007) Minimally invasive techniques for the management of lumbar disc herniation. Orthop Clin N Am 38: 363-372.

12. Yeung AT, Yeung CA (2006) In-vivo endoscopic visualization of patho-anatomy in painful degenerative conditions of the lumbar spine. Surg Technol Int 15 243-256.

13. Hirano Y, Mizuno J, Takeda M , Itoh Y, Matsuoka H, et al. (2012) Percutaneous endoscopic lumbar discectomy - early clinical experience. Neurol Med Chir (Tokyo) 52: 625-630. 
Citation: Hirano Y, Pawar S, Bansil R, Watanabe K (2018) Advantages of Endoscope-Assisted Disc-FX in Surgical Management of Lumbar Disc Herniation: A Report of 10 Cases. J Spine 7: 015. doi: 10.0142/2165-7939.1000015

14. Weinstein JN, Lurie JD, Tosteson TD, Tosteson ANA, Blood EA, et al. (2008) Surgical versus non-operative treatment for lumbar disc herniation: Four-year results of the Spine Patient Outcomes Research Trial (SPORT). Spine (Phila Pa 1976) 33: 2789-2800.

15. Gerges FJ, Lipsitz SR, Nedeljkovic SS (2010) A systematic review on the effectiveness of the Nucleoplasty procedure for discogenic pain. Pain Physician 13: $117-132$.

16. Hellinger S (2011) Disc-FX - A treatment for discal pain syndromes combining a manual and radiofrequency-assisted posterolateral microtubular decompressive nucleotomy. Eur Musculoskeletal Rev 6: 100-104.

17. Hellinger S, Liao X, Mermelstein P, Naresh K, Nucci R, et al. (2011) Radiofrequency-assisted lumbar semi-endoscopic manual discectomy using the Disc-FX system - Preliminary results of various ongoing clinical outcome studies worldwide. Eur Musculoskeletal Rev 6: 265-271.

18. Hellinger S (2014) Treatment of contained lumbar disc herniations using radiofrequency assisted micro-tubular decompression and nucleotomy: A four year prospective study results. Int J Spine Surg 8.
19. Kumar N, Kumar A Siddharth MS, Sambhav PS, Tan J (2014) Annulonucleoplasty using Disc-FX in the management of lumbar disc pathology. Int J Spine Surg 8.

20. Kumar N, Zaw AS, Kumar N, Sonawane D, Hey HWD, et al. (2018) Annulonucleoplasty using Disc-FX in the management of degenerative lumbar disc pathology: How long can the effect last? Global Spine J 8: 365-373.

21. Peul WC, Van den Hout WB, Brand R, Thomeer RTWM (2008) Prolonged conservative care versus early surgery in patients with sciatica caused by lumbar disc herniation: two year results of a randomized controlled trial. BMJ 336: $1355-1358$

22. Yeung AT (1998) Minimally invasive disc surgery with Yeung Endoscopic Spine System (YESS). Surg Technol Int 8: 267-277.

23. Yeung AT (2000) The evolution of percutaneous spinal endoscopy and discectomy: State of the art. Mt Sinai J Med 67: 327-332.

24. Beyaz SG, İnanmaz ME, Zengin EŞ, Ülgen AM (2016) Combined use of high radiofrequency disc ablation, annulus modulation, and manual nucleotomy in a patient with extruded disc herniation. Pain Pract 16: E74-E80.
This article was originally published in a special issue, Minimally Invasive Spine Surgery -II handled by Editor(s). Anthony T. Yeung, M.D. Clinical professor, University of New Mexico School of Medicine Associate Desert Institute for Spine Care, Phoenix, Arizona Executive director International Intradiscal therapy Society Phoenix, Arizona 